\title{
Die Polysemie des Verbes ziehen aus kognitiver Sicht
}

\section{Maria Jelisariewa}

The article deals with the multiple meaning of the German verb ziehen, previously studied in the works of W. Porzig, F. Hundsnurscher and G. Fritz, who worked at distinguishing only the selected meanings of a polysemant, not at studying the interrelation of the different meanings. In this article, the invariant of the verb ziehen is developed using 18 image-schemas, created after the study of the contextual examples taken from a German Text Corpus DWDS. Despite the fact that the image-schemas vary, they all represent the linear character of the denoted object form, motion trajectory or both, the latter can be regarded as the invariant of the verb ziehen, thus explaining its extreme polysemy

Polysemy - image-schema - invariant - corpus linguistics - movement verbs

Der Aufsatz will die Polysemie des Verbes ziehen aus kognitiver Sicht erörtern und setzt sich zum Ziel, anhand von Korpusbelegen ein kognitives Modell (Image-Schema) dieses Verbs zu entwickeln, das geeignet wäre, Zusammenhänge zwischen allen Bedeutungen ans Licht zu bringen. Anhand der Korpusbelege wurden 18 Image-Schemata skizziert, die Gemeinsamkeiten aufweisen: Linearität der Bewegungsbahn eines Gegenstandes, Linearität der Form des Gegenstandes oder beides. Die Idee der Linearität liegt allen Bedeutungen des Verbes ziehen zugrunde und kann als seine semantische Invariante gelten.

Polysemie - Image-Schema - Invariante - Korpuslinguistik - Bewegungsverben

\section{Einleitung}

Die Polysemie des Verbes ziehen hat bereits mehrfach die Aufmerksamkeit der Sprachwissenschaft auf sich gezogen, z. B. bei Porzig (1959), Hundsnurscher (1996) und Fritz (2000). Die genannten Studien waren allerdings allein auf die Analyse der Polysemie ausgerichtet, d. h. stellten Versuche dar, eine scharfe Trennungslinie zwischen unterschiedlichen Bedeutungen zu ziehen, während kaum Versuche unternommen wurden zu erklären, was alle Bedeutungen unter einem Sprachzeichen zusammenhält. Der Aufsatz will die Polysemie des Verbes ziehen aus kognitiver Sicht erörtern und setzt sich zum Ziel, anhand von Korpusbelegen ein einheitliches Image-Schema dieses Verbs zu entwickeln, das geeignet sein könnte, Zusammenhänge zwischen allen Bedeutungen aufzuzeigen.

\section{Polysemie als Forschungsgegenstand der Sprachwissenschaft}

Das Sprachphänomen Polysemie kommt in allen natürlichen Sprachen vor und stellt damit ein Kennzeichen der letzteren dar. Unter „Polysemie“ versteht man „die Fähigkeit des Wortes (einer Wortform), mehrere miteinander zusammenhängende Bedeutungen zu haben" (Olšanskij und Guseva 2005: 32, Übersetzung M. J.). Unter „Bedeutung des Wortes“ wird hier eine „geschichtlich bedingte Verbindung 
zwischen dem Lautkörper und der Abbildung des Gegenstands oder der Erscheinung in unserem Bewusstsein" (Beljaevskaja 1987: 22, Übersetzung M. J.) verstanden.

Dieser Zusammenhang zwischen den Bedeutungen entsteht meistens dadurch, dass der bestehende Lautkörper mit neuen Gegenständen, Erscheinungen oder Prozessen assoziiert wird, aufgrund der Ähnlichkeiten (Metapher) oder räumlichen, kausativen oder anderen Zusammenhängen (Metonymie), die ein menschlicher Betrachter festgestellt hat.

Je nach der Richtung der Bezeichnungsübertragung unterscheidet man drei Typen von Polysemie: radiale, kettenartige und kombinierte Polysemie (Krongauz 2005: 127). Bei radialer Polysemie werden alle Nebenbedeutungen von der Hauptbedeutung abgeleitet (z. B. bei dem Wort Pferd - im Sinne vom Tier, Turmgerät und Schachfigur), während bei der kettenartigen Polysemie jede weitere Bedeutung mit der vorigen verknüpft ist (wie z. B. beim Wort Film). Kombinierte Polysemie verbindet die beiden vorherigen Typen (z. B. beim Wort Fuchs).

Die Entwicklung der kognitiven Linguistik und der kognitiven Semantik als ihrer Abzweigung führte zur Entstehung der kognitiven Herangehensweise an die Polysemie (Lakoff 1987, Kubrjakova 2001, Evans, Green 2006, Pesina 2011, Leščëva 2014). Das Wort wird dabei als prototypische Kategorie betrachtet, dessen Mitglieder (d. h. die einzelnen Bedeutungen) ein gemeinsames konzeptuelles Schema (Prototyp) haben. Dieser Prototyp kann durch ein abstraktes Schema dargestellt werden und dient als Grundlage für die Entstehung von neuen Bedeutungen durch Metapher und Metonymie (Evans, Green 2006: 340). Die Anwendung von ImageSchemas wurde von Langacker (Langacker 1988) eingeführt mit dem Zweck, die zeitlich-räumliche Semantik durch Bilder mit Trajector (d. h. einem beweglichen Objekt) und Landmark (d. h. einem unbeweglichen Hintergrund) anschaulich zu machen. Image-Schemas eignen sich besonders gut für die Analyse von polysemen Raumpräpositionen und Raumpräfixen sowie von Bewegungsverben.

DiekognitiveHerangehensweisehat sich auch in der russischen Sprachwissenschaft durchgesetzt (Beljaevskaja 1987, Pesina 2011, Leščëva 2014). Dabei wird nicht selten der Begriff „semantische Invariante“ gebraucht. Darunter versteht man eine Gesamtheit von semantischen Merkmalen („Bedeutungskern“), die bei allen Bedeutungen des Polysemanten stabil und kontextunabhängig sind (Leščëva 2014: 10). Laut Beljajevskaja wird jeder neue Inhalt (d. h. ein neuer Gegenstand, eine neue Erscheinung oder ein neuer Prozess), für den eine Bezeichnung, ein Lautkörper gefunden werden muss, mit den bestehenden semantischen Invarianten (Prototypen) „verglichen“ und anschließend einer der Kategorien zugeordnet und entsprechend nominiert. 


\section{Polysemie des Verbes ziehen: Modelle von Porzig und Fritz}

Porzig ging bei der Analyse des Verbs ziehen davon aus, dass die Einheit eines mehrdeutigen Wortes „auf seiner Geschichte beruht“ und dass „die Zugehörigkeit zu ganz verschiedenen Wortfeldern“ (Porzig 1959: 163) für die Unterscheidung zweier oder mehrerer selbstständiger Wörter genügen müsse. Für das Verb ziehen bestimmt Porzig folgende inhaltliche Komponente: „eine Kraft, die zieht, etwas, das gezogen wird, eine Richtung, in der gezogen wird“ (ebd.). Porzig ist der Meinung, dass in den Beispielen einen Strich ziehen und Kaiser Rotbart zieht ins Heilige Land verschiedene Inhalte des Verbs ziehen vorliegen, was folgende Feldzusammenhänge bestätigen: Im ersten Fall sind dies die Verben schleppen, schieben, tragen und im zweiten - marschieren, wandern. Genauso verhalte es sich bei den Sätzen Sie sind nach Köln gezogen und Zieht es Ihnen?, weil es im ersten Fall um die Verlegung eines Wohnsitzes und im zweiten um eine (physische) Empfindung gehe. Mit anderen Worten vertritt W. Porzig die Ansicht, dass das Verb ziehen nicht mehrdeutig sei, sondern eine Gesamtheit verschiedener Wörter darstelle.

Fritz skizziert beim Verb ziehen zwei grundlegende Ereignistypen: „1) A übt auf einen Gegenstand (gegen einen Widerstand) eine Kraft in Richtung A hin aus mit der Intention, den Gegenstand zu A hinzubewegen; und 2) A übt auf einen Gegenstand (gegen einen Widerstand) eine Kraft in Richtung A hin aus, so dass sich der Gegenstand in der Bewegungsrichtung von A mitbewegt" (Fritz 2000: 40). Prototypische Beispiele seien Das Kind zieht am Türgriff; Das Kind zieht den Wagen. Ferner unternimmt Fritz einen Versuch, Zusammenhänge einiger etablierter Verwendungsweisen von ziehen mit den beiden grundlegenden Ereignistypen zu explizieren und beschreibt folgende „Muster“:

1) Das Extraktions-Muster (Er zieht den Korken aus der Flasche; Er zog den Colt aus dem Halfter; Der Zahnarzt zieht den Backenzahn; Die Pflanzen ziehen Nährstoffe aus dem Boden; Der Tee muss noch ziehen), hierzu gehören u.a. auch metaphorische Verwendungsweisen (Die Manager ziehen sich aus der Verantwortung; Er zieht den Kopf aus der Schlinge);

2) Das Verformungs-Muster, besonders bei elastischen Gegenständen ( $A$ zieht den Kaugummi, den Draht; die Plastikmasse lässt sich ziehen) sowie metaphorischen Übertragungen (Die Sitzung zieht sich in die Länge);

3) Das Magnet-Modell (Der Magnet zieht nicht mehr) sowie Metaphern (Viele Leute zieht es in die Südsee);

4) Kraftentfaltung (Der Motor zieht wie der Teufel);

5) Herstellung eines Gegenstands mit einer bestimmten Verlaufslinie (Der Nachbar zieht eine Mauer, einen Zaun (hoch), einen Graben);

6) Verlaufslinie eines Gegenstands (Der Fluss zieht sich durch die Ebene);

7) Bewegung in eine bestimmte Richtung (Die Wolken ziehen am Himmel, Die Schwalben ziehen nach Süden, Der Zirkus zieht von Gießen nach Wetzlar); 
8) Bewegung von flüchtigen Materien (A zieht den Rauch in die Lunge; Es zieht);

9) Metaphorische, idiomatische und isolierte Verwendungsweisen $(j-n$ über den Tisch ziehen, Fäden ziehen, in Erwägung ziehen).

Die oben angeführte Klassifikation von Fritz ist detailliert und sachlich, allerdings macht sie nicht deutlich, weshalb all diese Inhalte mithilfe eines Sprachzeichens ausgedrückt werden. Außerdem werden bei Fritz (2000) nicht alle verbreiteten Verwendungsweisen des Verbes ziehen und entsprechende Redewendungen erörtert bzw. erwähnt; zudem werden einige Phraseologismen angeführt, ohne ihren Bedeutungstyp zu nennen. Um diese Lücken zu füllen und die semantische Struktur des Verbes ziehen zu explizieren, ist es nötig, eine breitere Palette von Verwendungsweisen zu untersuchen, was anhand von Korpusbelegen möglich ist.

\section{Polysemie des Verbes ziehen: Image-Schemas als Darstellungsmittel}

Als Quelle für das Sprachmaterial diente das Digitale Wörterbuch der deutschen Sprache DWDS, und zwar das Kernkorpus des 20. Jahrhunderts (1945-2000) sowie das Kernkorpus des 21. Jahrhunderts (http://www.dwds.de/?qu=ziehen). Im Laufe der Untersuchung wurden Belege mit dem Verb ziehen aus den beiden Korpora ausgesondert und in mehrere Gruppen eingeteilt, je nachdem, mithilfe welchen Image-Schemas sich ihre Bedeutungen darstellen lassen, wobei ein ImageSchema mehrere Bedeutungen überdachen kann. Anhand der Korpusbelege wurden 18 Image-Schemata skizziert. Diese dienen fast ausnahmslos zur Beschreibung von mehreren Bedeutungen und erklären eine Anzahl von Redewendungen und festen Wortverbindungen.

Die Beschreibung des ersten Schemas (Abb. 1) kann folgenderweise sprachlich ausgedrückt werden: «Ein Gegenstand bewegt sich und übt auf einen anderen Gegenstand Kraft so aus, dass der letztere mitbewegt wird, wobei die Bewegungsbahn eine Linie bildet». Dieses Schema überdacht mehrere Bedeutungen von ziehen, die der entsprechende Artikel im Duden auflistet (http://www.duden. de/rechtschreibung/ziehen): 1) hinter sich her in der eigenen Bewegungsrichtung in gleichmäßiger Bewegung fortbewegen; 2) jemanden [an der Hand] mit sich fortbewegen; 3) durch Einschlagen des Lenkrades, Betätigen des Steuerknüppels in eine bestimmte Richtung steuern. Dieses Schema kann durch die folgenden Korpusbelege illustriert werden:

(1) An diesem Sonntag wandte er sich mal wieder mir zu, zog mich in die Küche, wo Frau Fuchs saß und auf weitere Instruktionen wartete. (Frank Goosen, Liegen lernen).

(2) Er trug zuletzt einen zerrissenen Frack, zog eine Karre durch die Stadt, sammelte krumme Nägel, die er entrostete und auf einer alten Drehbank richtete, in seiner Bude am Bahndamm hinter Ginstergestrüpp, ein Areal, das die Familie dann 
kaufte und unter Landschaftsschutz stellen ließ. (Degenhardt, Franz Josef, Für ewig und drei Tage).

(3) Rammins Pferde, von ihm in Richtung von Doras Wirtschaftsgarten in Gang gesetzt, begriffen auch ohne Zügelhilfe, daß sie nur in einer halben Wendung aus diesem Hof kommen würden, und zogen den Wagen fürsorglich auf die Ausfahrt. (Johnson, Uwe, Jahrestage).

(4) Jarvens Mutter zog den Wagen mit hoher Geschwindigkeit nach rechts, dann auf die Ausfahrt. (Kirsten Boie, Skogland).

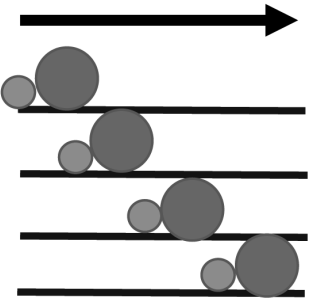

Abb. 1

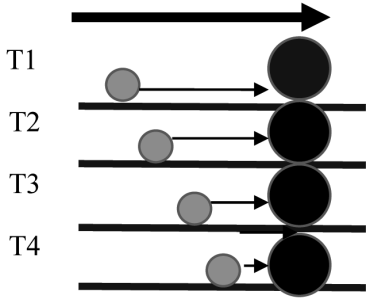

Abb. 2

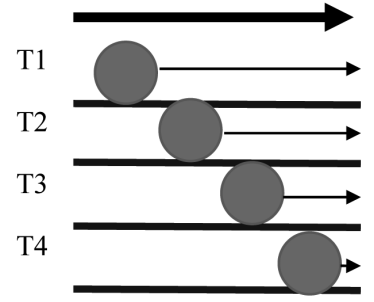

Abb. 3

Die Bedeutung von ziehen im Beispiel (4) sowie im Beispiel von G. Fritz Der Motor zieht wie der Teufel kann man als historisch bedingte Modifikation von Bedeutung (3) betrachten: Ursprünglich waren es Tiere, die Verkehrsmittel in Bewegung setzten, später wurden sie aber durch Autos mit Motor ersetzt und der Mensch am Steuer begann die Richtung zu bestimmen, in die das Auto fährt. Obwohl sich die Komponenten, die diese Art von Fortbewegung bestimmen, verändert haben, wird diese weiterhin sprachlich identisch ausgedrückt.

Dasselbe Image-Schema liegt z. B. den folgenden Redewendungen zugrunde: etwas nach sich ziehen, Konsequenzen ziehen, durch den Dreck ziehen, ins Gespräch ziehen, vor den Kadi ziehen.

Das zweite Image-Schema muss als grundlegend betrachtet werden (Abb. 2): «Ein bewegungsloser Gegenstand übt auf einen anderen Gegenstand Kraft aus, die bewirkt, dass sich dieser nähert, wobei die Bewegungsbahn eine Linie bildet». Diesem Image-Schema entsprechen zwei Bedeutungen aus dem Duden: 1) in gleichmäßiger Bewegung [über den Boden oder eine Fläche] zu sich her bewegen und 2) Anziehungskraft haben. Das Schema wird durch die folgenden Korpusbelege veranschaulicht:

(5) Nie hätte ich erwartet, dass er mich heftig an sich zog und in meinen Armen schluchzte wie ein kleines Kind. (Ingrid Noll, Ladylike).

(6) Zur Frage einer neutralen Haltung sagte er, daß ein neutralisiertes Deutschland sich dann zwischen einer mächtigen Sowjetunion und einem schwachen Europa befinden und Gefahr laufen würde, vom stärkeren Magneten in sein Kraftfeld gezogen zu werden. (Archiv der Gegenwart, 20, 1950, Außenpolitik. Wehrwesen. Parteien. ATLANTIKPAKT [11.12.50]). 
Das zweite Schema erklärt metaphorische Redewendungen wie Blicke, Hass, Kritik auf sich ziehen, sowie jemanden auf seine Seite ziehen.

Das dritte Image-Schema (Abb.3) stellt eigentlich eine Variation des zweiten dar, aber der Gegenstand, der etwas zu sich herbewegt, wird sprachlich als eine Kraft angegeben oder wird nicht explizit benannt. Stattdessen gibt man die Bewegungsrichtung oder den Endpunkt an:

(7) Da sind wir«, sagte der Stollentroll. Er steckte seinen Zeigefinger in ein kleines unscheinbares Loch neben der Tür, und sie öffnete sich ohne jedes Geräusch wie ein Theatervorhang, der in die Höhe gezogen wird. (Moers, Walter, Die 13 1/2 Leben des Käpt'n Blaubär).

(8) Ich ziehe zurück wie vom Feuer, und eine geheime Kraft zieht mich wieder vorwärts - mir wird's so schwindelig von allen Sinnen. (Bädekerl, Klaus, Alles über Geld und Liebe).

Damit hängt die metaphorische Verwendungsweise mit dem Pronomen es zusammen:

(9) Groot und Zille zog es nach Buchting, um von dort aus in See zu stechen. Sie wollten andere Kontinente kennenlernen, "unseren Horizont erweitern", wie Zille es ausdrückte. (Moers, Walter, Die 13 1/2 Leben des Käpt'n Blaubär).

Auf diesem Schema beruhen feste Redewendungen wie zur Rechenschaft, zur Verantwortung, zu Rate ziehen, einige abstrakte Phraseologismen mit der Präposition in (ins Lächerliche, in Betracht, in seinen Bann, in Kalkül, in Zweifel, in Erwägung, in Mitleidenschaft, ins Vertrauen ziehen), sowie einige Redewendungen, die auf einem konkreten bildlichen Ausdruck basieren: in den Abgrund, in den Schmutz ziehen.

Das vierte Image-Schema umfasst eine reiche Palette von Situationen (Abb. 4): "Der Mensch bewegt einen Gegenstand so, dass seine Bewegungsbahn eine Linie bildet». Dabei kann die Bedeutung des Verbes ziehen unterschiedlich sein (waagrechte Pfeile bezeichnen die ausgeübte Kraft, senkrechte Pfeile die Bewegungsbahn).

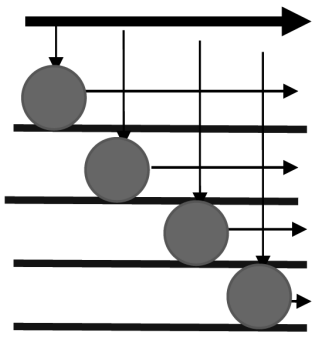

Abb. 4

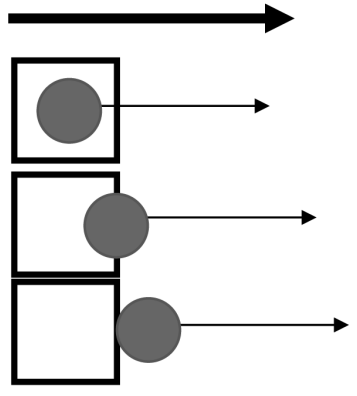

Abb. 5

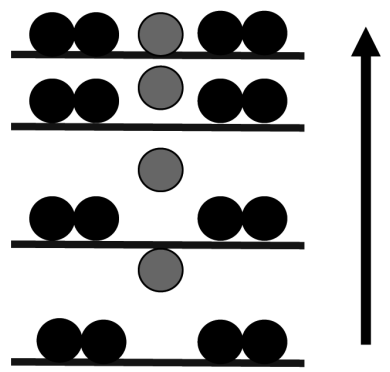

Abb. 6 
(10) Als ehemaliger Bewunderer kam ich nicht umbin, innerlich den Hut vor ihm zu ziehen. (Moers, Walter, Die 13 1/2 Leben des Käpt'n Blaubär).

(11) Okay, machen wir", sagte Benno Kröttmann, stellte die Flamme wieder heller und heißer und zog die Schutzbrille vor die Augen. (Degenhardt, Franz Josef, Für ewig und drei Tage).

(12) Wolfgang Hess aus Langen, ein großer, kräftiger Mann, bekam eine Whiskyflasche über den Kopf gezogen, und knapp am Kopf von Nero Reisz flog ein $C D$-Player vorbei. (Hasselbach, Ingo/Bonengel, Winfried, Die Abrechnung).

(13) Sie zog die Karte durch ibren Apparat und runzelte die Stirn. (Frank Goosen, Liegen lernen).

(14) Herodias zieht ihm den Ring vom Finger und lässt ihn dem Henker zum Zeichen der Hinrichtung überreichen. (Fath, Rolf, Reclams Lexikon der Opernwelt Band 5).

(15) Wenn Weißzuletzt den Turm gezogen hätte, wäre der schwarze König bereits durch den Läufer im Schach gewesen; wenn er den Läufer gezogen hätte, durch den Turm (Der Spiegel 09.08.1982).

(16) Basta zog die Flinte von der Schulter, blieb stehen und lauschte. (Cornelia Funke, Tintenherz).

Als sehr produktiv erweist sich das fünfte Schema «Extraktion aus einem Behälter» (Abb. 5), bei dem wieder die Bewegungsbahn eine Linie bildet. Durch das Schema werden zwei Bedeutungen darstellt: 1) mit einer ziehenden Bewegung aus etwas herausnehmen, herausholen und 2) gewinnen (z.B. Öl aus Pflanzen ziehen):

(17) Basta stießStaubfinger so grob von sich, dass er hinfiel, und wollte sein Schwert ziehen, doch seine Hände, bleich wie Papier hatten offenbar noch keine Kraft. (Cornelia Funke, Tintenherz).

(18) Dann habe er plötzlich eine Waffe gezogen und mehrere tödliche Schüsse auf den König abgegeben. (Ermordung von König Faisal; Kronprinz Khaled neuer König; Prinz Fahd neuer Kronprinz).

(19) Den Weisheitszahn, den er ziehen mußte, gab er mir hinterher mit und erklärte, wenn ich den bei Sonnenuntergang in den Nil werfen würde, bekäme ich mein Lebtag kein Zahnweh mehr. (Maltzan, Maria von, Schlage die Trommel und fürchte dich nicht).

(20) Nachts nicht, sagt der Arzt; am besten wäre, Sie legen sich mal eine Woche ins Krankenhaus und lassen sich die verbrauchten Krampfadern ziehen. (Wilhelm Genazino, Die Liebesblödigkeit).

(21) Er hatte ein Formular aus der Schublade gezogen und legte es auf den Tisch. (Moers, Walter, Die 13 1/2 Leben des Käpt'n Blaubär).

Dieses Schema erklärt zahlreiche abstrakte metaphorische Redewendungen wie z. B. Fazit, Schlüsse, Schlussfolgerungen, Profit, Nutzen, Vorteil, Gewinn, Lehren aus etwas ziehen, jemandem etwas aus der Nase ziehen. Außerdem kann den Redewendungen auch ein konkretes visuelles Bild zugrunde liegen: die Wurzel aus 
einer Zahl ziehen (wie wenn man eine Pflanzenwurzel aus der Erde zieht), seinen Kopf aus der Schlinge ziehen, den Karren aus dem Dreck ziehen, etwas ans Tageslicht ziehen, einer Sache den Zahn ziehen.

Eine Variation dieses Schemas ist das nächste, sechste Schema «aus einer bestimmten Menge auswählen und herausholen» (Abb. 6): ein Buch aus dem Regal ziehen, eine Blume aus der Vase ziehen. Das Schema erklärt auf einem Bild basierende Redewendungen wie ein Los, die Arschkarte, eine Niete, den Kürzeren ziehen.

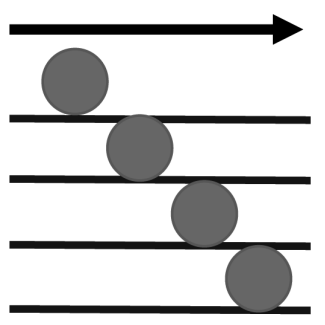

Abb. 7

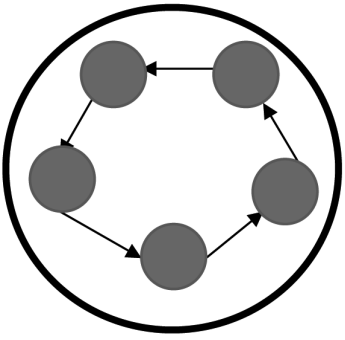

Abb. 8

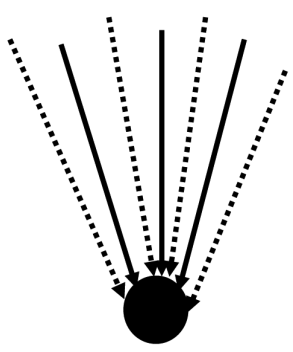

Abb.9

Die nächste Gruppe von Schemata unterscheidet sich von den oben genannten dadurch, dass sie Situationen beschreibt, in denen es nur einen Gegenstand gibt, der sich bewegt und dessen Bewegungsbahn auch linear ist. Es gibt kein passives Objekt, das gezogen wird. Hier sind drei Image-Schemata zu unterscheiden. Das siebte Schema (Abb. 7) stellt zwei Bedeutungen von ziehen dar: 1) seinen [Wohn-] Sitz irgendwohin verlegen, umziehen; 2) sich stetig fortbewegen, irgendwo[hin] bewegen; irgendwo[hin] unterwegs sein:

(22) Helene Amalie Ascherfeld war die Enkelin eines reformierten Kaufmanns, der Ende des 17. Jahrhunderts nach Essen gezogen und rasch zu erheblichem Wohlstand gelangt war. (Lothar Gall, Krupp).

(23) Willy Brandt sei ja dem Generalsekretär gut bekannt. Er ziehe jetzt wie ein alter Bischof oder Metropolit durch die DDR, mit segnender Hand und als Ehrenvorsitzender der SPD. (Nr. 174: Gespräch Kohl mit Gorbatschow vom 10. Februar 1990).

(24) Mir schwanden die Sinne, mir wurde schwarz vor den Augen, mir wurde lila und grün, dann sah ich Möwen, Schwäne und Kraniche ziehen. (Ingo Schulze, Neue Leben).

(25) Noch 1962 aber ärgerte Edmund Wilson der Vorwand, unter dem die Deutschen 1914 in den Krieg gezogen waren. (Wolf Lepenies, Kultur und Politik).

Das achte Schema (Abb. 8) drückt die Bedeutung «sich kreisend bewegen» aus, die es aber im Duden als selbstständige Bedeutung nicht gibt: 
(24) Ich breitete die Arme aus - und ich flog! Ich stürzte nicht mehr, sondern zog wie ein Adler majestätische, langsame Kreise, trudelte in einer weiten Spirale nach unten. (Moers, Walter, Die 13 1/2 Leben des Käpt'n Blaubär).

(25) Wenn die Formel-1-Boliden ihre Runden ziehen, versammeln sich weltweit bis zu einer Milliarde Fans vor der Glotze. (Die Zeit 30.08.1996).

Die Situation, die durch das neunte Image-Schema (Abb. 9) repräsentiert wird, kommt im Duden auch nicht als selbständige Bedeutung vor: «Menschen oder Tiere bewegen sich in Richtung eines (großen) Gegenstands so, dass ihre Bewegungsbahnen Linien bilden»:

(26) Nach Schlußdieser Veranstaltung zogeine kleine Gruppe von Demonstranten zum Hauptbahnhof, um dort den Protest fortzusetzen. (Hannover, Heinrich, Die Republik vor Gericht 1954-1974).

(27) Da Fliegen zum Licht ziehen, kam nach ein paar Augenblicken der Geheimdienstler als brummende Fliege ins Zimmer geflogen. (Herta Müller, Der König verneigt sich und tötet).

Die Gruppe von Bedeutungen, die von G. Fritz als Bewegung von flüchtiger Materie bezeichnet wurde, lässt sich weiter differenzieren, indem man sie in drei Schemata unterteilt (Abb. 10-12): 1) Luftmassen bewegen sich linear nach innen (frische Luft in die Nase ziehen, an der Zigarette ziehen, der Ofen zieht gut); 2) ein Geruch verbreitet sich in einer bestimmten Richtung (Der Duft von Holunderblüten zog durch das Zimmer); 3) Luftmassen bewegen sich linear, sodass ihre Bewegung spürbar wird (Es zieht, wer macht das Fenster auf?).

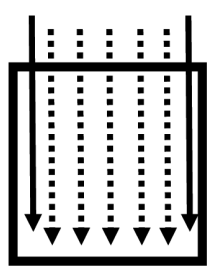

Abb. 10

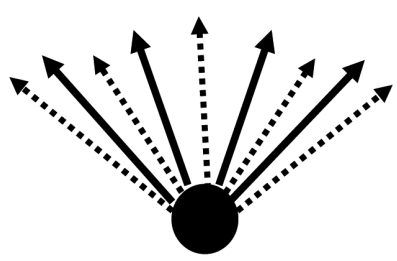

Abb. 11

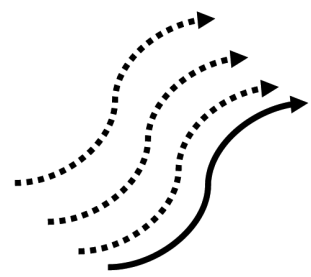

Abb. 12

Weitere Image-Schemas legen den Schwerpunkt nicht auf die Linearität der Bewegung, sondern auf die Linearität der Form des Gegenstandes: «Ein Subjekt erzeugt einen anderen Gegenstand, dessen Form linear ist» (Abb. 13):

(28) Zwischendurch frisiert er das Haar nach vorne und ziebt an beliebigen Stellen einen Scheitel. (Wilhelm Genazino, Die Liebesblödigkeit).

(29) Früher bin ich mit den Kindern manchmal hier essen gegangen, für 2,50 Mark pro Person. Dann hat die Stasi es für sich entdeckt und einen Giftzaun um das Anwesen gezogen. (Die Zeit 15.07.1999). 
(30) Lisa huschte ins Bad, zog sich einen Strich um die Augen, tupfte etwas Parfüm an den Hals, ordnete die Haare, zupfte ihr T-Shirt zurecht und öfnete einen Knopfmehr. (Jentzsch, Kerstin, Seit die Götter ratlos sind).

Auf diesem Schema beruhen Redewendungen wie einen Schlussstrich, eine Trennlinie, einen Trennungsstrich, eine Grenze, Parallelen ziehen, wie mit dem Lineal gezogen sein, sowie weniger offensichtliche seine Bahn ziehen, Vergleiche ziehen. $\mathrm{Zu}$ einem bestimmten Grade entsprechen diesem Schema auch Verwendungsweisen von ziehen bei der Beschreibung von Mimik: eine Grimasse ziehen, ein langes Gesicht ziehen, die Mundwinkel nach unten ziehen, die Stirn in Falten, kraus ziehen, die Lippen zur Seite ziehen.

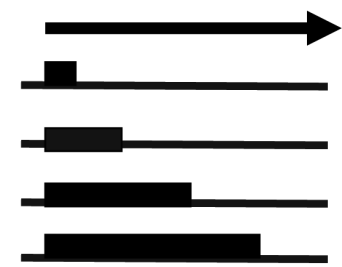

Abb. 13

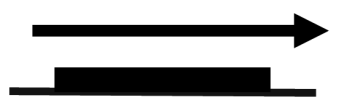

Abb. 14

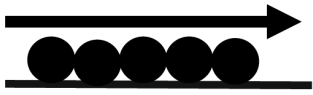

Abb. 15

Das 14. und 15. Schema beschreibt auch die Statik und ist mit dem 13. Schema verwandt. Das 14. Schema (Abb. 14) stellt folgende Bedeutungen dar: 1) sich [auf irgendeine Weise] irgendwohin erstrecken, bis irgendwohin verlaufen und 2) sich hinziehen.

(31) Um die kaiserlichen Teegärten zogen sich hohe Mauern, und die Teepflücker, die zur Erntezeit gerufen wurden, standen schon bei den kleinsten "Vergehen" unter strengen Strafen. (o.A., Die grosse Welt der Getränke).

(32) Er trägt einen roten Trainingsanzug von Adidas. Die drei Streifen ziehen sich über die gesamte Fläche. (Lebert, Benjamin, Crazy).

(33) Es läuft nämlich im Augenblick der dritte Akt in einem Drama (oder einer Posse), das sich seit sieben Jahren hinzieht und sich wohl noch länger ziehen wird. (Der Spiegel 18.01.1988).

Die Tatsache, dass das Verb ziehen für die Beschreibung von Zeit (ins Unendliche ziehen, sich in die Länge ziehen) verwendet wird, wird durch die konzeptuelle Metapher ZEIT IST RAUM erklärt, die für alle indogermanischen Sprachen kennzeichnend ist.

Weniger verbreitet ist das 15 . Schema, das wie folgt lautet: «Mehrere Gegenstände bilden zusammen eine reale oder vermeintliche Linie " (Abb. 15).

(34) Wir wuchsen ja auf im Schatten der endlosen Russenkasernen, die sich von Klotzsche bis hinein in die Stadt zogen. (Ingo Schulze, Neue Leben). 
(35) Einer von ihnen, schwarz wie Ruß, kreist 27400 Kilometer über den Neptunwolken, der andere, 37500 Kilometer entfernt, besteht nur aus Ringbruchstücken, die sich als gekrümmte Bänder um den Neptun-Äquator ziehen. (Der Spiegel 28.08.1989).

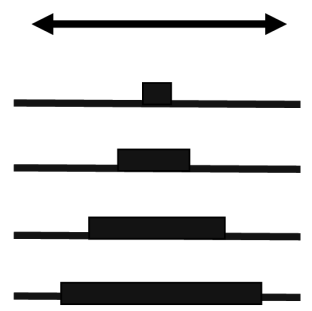

Abb. 16

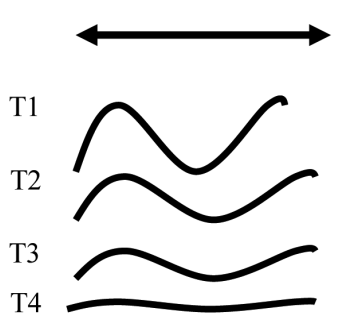

Abb. 17

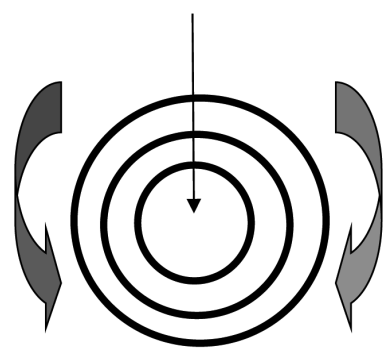

Abb. 18

Die letzten drei Image-Schemata stellen die Verformung oder Bewegung eines Gegenstands dar, dessen Form linear ist. Der Gegenstand kann durch Dehnung verlängert (Abb. 16), gestreckt oder fortbewegt werden (Abb. 17). Im ersten Fall, z. B. Kaugummi ziehen, der Teig zieht Fäden, in übertragener Bedeutung - Laute, Töne, Silben ziehen, im zweiten Fall - Draht, eine Leitung ziehen, den Faden durchs Nadelöhr ziehen. Es lässt sich vermuten, dass die Bedeutung ,aufziehen, züchten ${ }^{*}$ (Rosen, Spargel, Schweine ziehen) ebenfalls durch das 16. Schema motiviert ist. Verformung eines Gegenstands kann auch dadurch eintreten, dass der Kreis, den dieser Gegenstand durch seine Form bildet, enger wird (Abb. 18):

(36) Sie hatten einen Kreis um ihn gebildet, zwanzig, dreißig Personen und zogen diesen Kreis nun enger und enger. (Süskind, Patrick, Das Parfum).

(37) Schließlich ist er doch gut erzogen", sie lachte und zog das seidene Tuch fester um ibren Hals. (Julia Franck, Lagerfeuer).

(38) Bolström zog den Gürtel seines Morgenmantels fester und setzte sich auf einen Stubl am Fenster. (Kirsten Boie, Skogland).

\section{Zusammenfassung}

An dieser Stelle lässt sich Folgendes zusammenfassen: Abgesehen davon, dass die hier entwickelten Modelle unterschiedlich sind, enthalten sie alle ein gemeinsames Element: Entweder stellt die Bewegungsbahn des Subjekts eine Linie dar (seine Bahn ziehen, Kreise ziehen) oder ihre Form ähnelt einer Linie (eine Linie, einen Strich ziehen). Dabei können sowohl die Bahn als auch die Form durch Linearität geprägt sein: einen Draht, eine Leitung ziehen, den Faden durchs Nadelöhr ziehen. Einige Schemata überdachen mehrere Wörterbuchbedeutungen, konkrete sowie übertragene, u. a. auch metaphorische (in Betracht ziehen, Konsequenzen ziehen, 
Kritik auf sich ziehen, in Kalkül ziehen, Nutzen ziehen, eine Niete ziehen usw.). Der Grundgedanke der Linearität liegt allen Bedeutungen des Verbes ziehen zugrunde und kann als seine semantische Invariante gelten, die sich in konkreten Varianten realisiert. Da diese Invariante ziemlich abstrakt ist und demzufolge auf viele extralinguistische Situationen angewandt werden kann, lässt sich vermuten, dass sich die extreme Polysemie des Verbes ziehen auch weiterentwickeln kann.

\section{Literaturverzeichnis}

Beljaevskaja, Elena (1987): Semantika slova (Semantik des Wortes). Moskau, Vysšaja škola.

Evans, Vyvyan und Green, Melanie (2006): Cognitive Linguistics - an Introduction. Edinburgh, Edinbourgh univversity press.

Fritz, Gerhardt (2000): Extreme Polysemie - der Fall ziehen. In: Beckmann. Susanne (Hg.): Sprachspiel und Bedeutung. Tübingen, S. 37-49.

Hundsnurscher, Franz (1996): Wortsemantik aus der Sicht einer Satzsemantik. In: Hundsnurscher, F. / Weigand, E. (eds.): Lexical Structures and Language Use. Proceedings of the International Conference on Lexicology and Lexical Semantics, Münster, September 13-15, 1994. In collaboration with Eckhard Hauenherm. Tübingen, Niemeyer. S. 39-51. Krongauz, Maxim (2005): Semantika (Die Semantik). Moskau, Izdatelskij Centr „Akademia“. Kubrjakova, Elena (2001): Tipy jazykovych značenij. Semantika proizvodnogo slova (Typen der Sprachbedeutungen. Semantik der Derivativen). Moskau, Izdatelstvo „Nauka“.

Lakoff, George (1987): Women, Fire and Dangerous Things: What Categories Reveal about the Mind. London; Chicago, The University of Chicago Press.

Langacker, Robert (1988): Foundations of cognitive grammar. Stanford/California, Stanford University Press.

Leščëva, Ljudmila (2014): Leksičeskaja polisemija v kognitivnom aspekte (Lexikalische Polysemie in kognitiver Sicht). Moskau, Jazyki slavjanskoj kultury.

Olšanskij Igor`/ Guseva, Alla (2005): Lexikologie: Die deutsche Gegenwartssprache. Moskau, Izdatelskij Zentr „Akademia“.

Pesina, Svetlana (2011): Slovo v kognitivnom aspekte (Das Wort in kognitiver Sicht). Moskau, FLINTA: Nauka.

Porzig, Walter (1959): Die Einheit des Wortes. In: Gipper, Helmut (Hg.), Sprache. Schlüssel zur Welt. Festschrift für Leo Weisgerber. Düsseldorf, Pedagogischer Verlag Schwann, S. 158-167.

Das Digitale Wörterbuch der deutschen Sprache (DWDS). http://www.dwds.de/?qu=ziehen (5.4.2015)

Worterbuch der deutschen Sprache. Duden.de. http://www.duden.de/rechtschreibung/ziehen (5.4.2015)

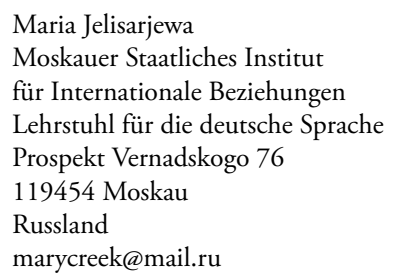

